\title{
Pericardial Tamponade And Lung Adenocarcinoma: Case Report And Literature Review
}

\author{
I. Lozano-Díez, M. López-Rodríguez, L. Cagide-González, and J. A. Díaz-Peromingo
}

\begin{abstract}
Pericardial effusion is the accumulation of fluid between the layers of the pericardium. I massive, pericardial tamponade and compression of the myocardium are life threatening conditions. The causes of pericardial effusion are varied, from idiopathic, neoplasms, iatrogenesis, and autoimmune. Pericardial tamponade can be a complication of neoplastic disease. Malignancy must be ruled out in every cardiac tamponade. Malignant etiology must be considered in patients with previous history of malignancy, pericarditis that does not respond to anti-inflammatory treatment, pericardial effusion that increases its amount rapidly, or recurrent pericardial effusion. Metastatic pericardial effusion due to lung cancer is not rare but not all lung cancers involve the same way the pericardium. In this paper, we present the case of a previously healthy patient with pericardial tamponade as presentation form of a lung adenocarcinoma and review the literature.
\end{abstract}

Index Terms - malignant pericadial effusion; cardiac tamponade; lung adenocarcinoma.

\section{INTRODUCTION}

Pericardial effusion is defined as the accumulation of more than $50 \mathrm{ml}$ of fluid between the visceral and parietal layers of the pericardium. The production of liquid exceeds the absorption capacity and, in the event of massive accumulation of liquid, we will be faced with pericardial tamponade with the consequent compression of the myocardium. In clinical practice it is a medical emergency that requires acting almost immediately to decompress the heart. The causes of pericardial effusion are very varied. In our setting, the most frequent cause is idiopathic (approximately half of the cases) followed by neoplasms (especially effusion secondary to pericardial metastasis), infections, iatrogenesis, and autoimmune etiology [1].

Clinical presentation of pericardial tamponade is diverse. Signs and symptoms are also very varied, from syncope, dyspnea, palpitations, precordial pain, electrocardiographic alterations, presence of paradoxical pulse, jugular venous engorgement, etc. Pericardial tamponade is a complication in $15 \%$ of patients with idiopathic pericarditis and can be as high as $60 \%$ in cases of neoplastic or infectious involvement [2].

In some cases, pericardial effusion may be the first manifestation of a neoplastic disease. For this reason, malignancy must be ruled out in those cases that debut with a

Published on June 6, 2020.

I. Lozano-Díez, Complejo Hospitalario Universitario de Santiago, Spain. (corresponding e-mail: irene.lozano.diez @ sergas.es)

M. López-Rodríguez, Complejo Hospitalario Universitario de Santiago, Spain.

(corresponding e-mail: maria.lopez.rodriguez ${ }^{\circledR}$ sergas.es) cardiac tamponade. Malignant etiology must be considered in patients with previous history of malignancy, pericarditis that does not respond to anti-inflammatory treatment, pericardial effusion that increases its amount rapidly, or recurrent pericardial effusion [3], [4].

We present the case of a previously healthy woman with pericardial tamponade as presentation form of a lung adenocarcinoma, reviewing the current literature in terms of malignancy and especially lung cancer as etiology.

\section{Clinical CASE}

A 69-year-old woman with no toxic habits or known vascular risk factors or other pathological history of interest was admitted to the emergency room for presenting continuous and progressive chest tightness during the last four days. Physical examination showed the presence of jugular venous engorgement at $45^{\circ}$ and arrhythmic tachycardia at $80 \mathrm{bpm}$. Blood pressure, temperature, pulmonary auscultation, and the rest of the general physical examination were normal. The EKG showed the presence of unknown atrial fibrillation and generalized low voltage. Chest radiography disclosed cardiomegaly and loss of volume in the right upper lobe as a casual discovery (Fig. 1). Blood count, coagulation study and blood biochemistry tests were normal. Given these findings, an echocardiogram was performed showing a severe circumferential pericardial effusion of up to $25 \mathrm{~mm}$ without signs of atrial or ventricular collapse. Hospitalization was decided to complete study and clinical follow-up. After a few hours, the patient presented abrupt clinical worsening with intense dyspnea, hypotension, and increase of intrapericardial fluid when the echocardiographic examination was repeated. Diagnostic and evacuating pericardiocentesis was performed. A total of $800 \mathrm{cc}$ of serohematic exudate with negative culture, negative auramine and a positive cytological study for adenocarcinoma was obtained. CT scanning was performed showing the presence of pericardial effusion, bilateral pleural effusion, low amount of ascites, and a mass in the right upper lobe with multiple mediastinal adenopathy (Fig. 2). Bronchoscopic examination revealed the presence of a stenosis affecting the anterior segment of the right upper lobe. Biopsy showed the presence of lung adenocarcinoma. After stablishing the diagnosis of metastatic lung adenocarcinoma, treatment with pemetrexed, carboplatin, and pembrolizumab

L. Cagide-González, Complejo Hospitalario Universitario de Santiago, Spain.

(corresponding e-mail: laia.cagide.gonzalez ${ }^{\circledR}$ sergas.es)

J. A. Díaz-Peromingo, Complejo Hospitalario Universitario de Santiago, Spain.

(corresponding e-mail: jose.antonio.diaz.peromingo@ ${ }^{@}$ sergas.es) 
was started with good tolerance after 8 cycles, and without recurrence of the pericardial effusion after 6 months of follow-up.

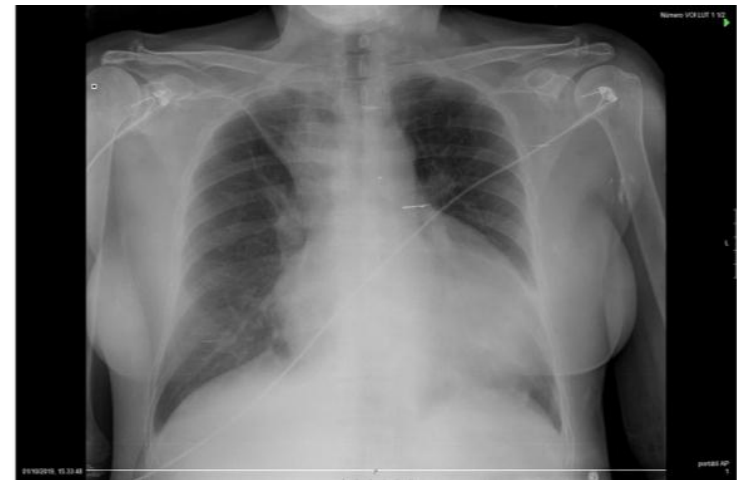

Fig. 1. Frontal chest radiograph showing mild cardiomegaly and opacification affecting the right upper lobe (arrow).

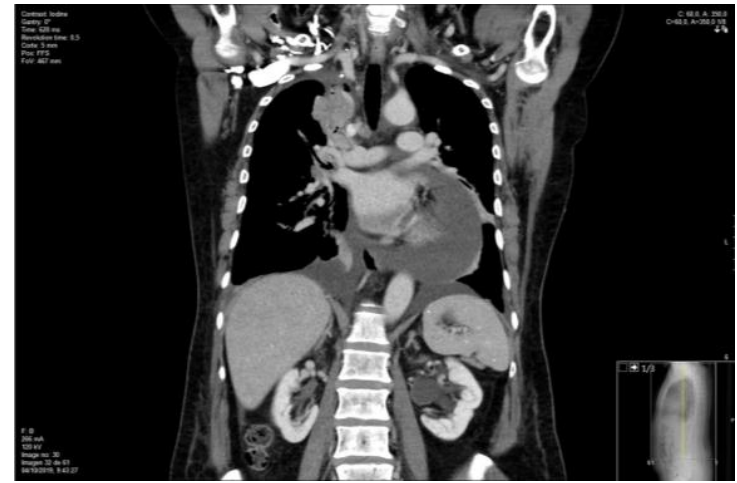

Fig. 2. CT scan imaging showing the presence of a severe pericardial effusion (arrow heads), and a mass affecting the right upper lobe bronchi (arrow).

\section{DISCUSSION}

Pericardial involvement is a relatively frequent manifestation in patients with known advanced malignancy. About 10-20\% of all cancers can develop some type of pericardium involvement (acute pericarditis, pericardial effusion, tamponade ...) [5], [6].

Metastatic involvement through blood or lymphatic vessels is the most common mechanism by which a primary tumor causes involvement of the pericardium. The most frequent solid tumors are lung in $40 \%$ (specifically non-small cell types, the first of which is frequently the adenocarcinoma type followed by squamous), breast $20 \%$, melanoma (high rate of cardiac metastases) and $7 \%$ gastrointestinal adenocarcinomas (esophagus, colorectal, and gastric) [7] [11]. There is a possibility, especially in lung tumors located in lobes close to the heart, that the pericardium is affected by direct invasion rather than by hematogenous spread like probably in our patient [3], [6], [12].

Hematologic tumors are another important group of nonsolid tumors that are relatively frequently associated with pericardial effusion, specifically both Hodgkin and nonHodgkin lymphomas and leukemias. It is estimated that up to $10 \%$ of pericardial effusions can be of hematological origin [5] - [7]. Other tumors less frequently associated with pericardial effusion are Kaposi's sarcoma, kidney and bladder tumors, thymoma, Ewing's sarcoma, adenocarcinoma of the endometrium, cervix, or ovary, and papillary thyroid carcinoma [3], [5], [13] - [15]. Finally, and much less frequently, there are primary tumors originating in the pericardium itself, both benign (myxomas $75 \%$, lipomas) and malignant types. Among malignancies, mesotheliomas characteristically form multiple masses at the level of the pericardium, and the appearance of pericardial effusion and even cardiac tamponade is frequent. Other less frequent pericardial tumors are fibrosarcomas, lymphangiomas, hemangiomas, and neurofibromas [3], [5] - [7], [16].

Furthermore, antineoplastic therapy (chemotherapy and radiotherapy) can cause pericardial involvement and therefore associated pericardial effusion. Specifically, in the case of radiotherapy, this affectation can manifest itself just a few weeks or even years after starting treatment [3], [5] - [7].

Regarding to lung cancer, pericardial effusion as clinical presentation is rare, and is associated with a worse prognosis. According to the different histological types of the disease, pericardial effusion does not occur with the same frequency in the different types. Lung adenocarcinoma is the most frequent histological type of lung cancer today, both in smokers and non-smokers with similar incidence in both sexes. Lung adenocarcinoma usually presents as a peripheral nodule and characteristically affects the pleura, being the first cause of malignant pleural effusion. Likewise, it is the lung cancer that most frequently presents pericardial effusion [17]. Lung cancer, more specifically adenocarcinoma, has been found to be the most common tumor cause of metastasis and malignant pericardial effusion after other non-lung tumors such as mesothelioma and melanoma. It has been observed that the lymphatic route is the most frequent dissemination route in relation to adenocarcinoma, above the hematological [18]. Anaplastic small cell carcinoma, which usually presents as a central mass is frequently associated with paraneoplastic syndromes, and squamous cell carcinoma, which usually presents as a cavitated mass and is associated with Pancoast syndrome, are the following types of lung cancer in frequency in which malignant pericardial effusion is a form of clinical presentation [17], [19]. Cases of malignant pericardial effusion have also been described in bronchoalveolar carcinomas, but less frequently than in previous histological types [17].

In short, although lung cancer is not the most frequent tumor cause of pericardial effusion, it should be considered in the differential diagnosis of patients with serohematic effusion as a cause of cardiac tamponade. In these cases, lung adenocarcinoma is the most frequent histological type.

\section{REFERENCES}

[1] A. D. Ristić, M. Imazio, Y. Adler, A. Anastasakis, L. P. Badano, A Brucato, et al., "Triage strategy for urgent management of cardiac tamponade: a position statement of the European Society of Cardiology Working Group on Myocardial and Pericardial Diseases", Eur Heart J., vol. 35, pp. 2279-2284, 2014.

[2] M. H. Khandaker, R. E. Espinosa, R. A. Nishimura, L. J. Sinak, S. N. Hayes, R. M. Melduni, J. K. Oh, "Pericardial disease: diagnosis and management", Mayo Clin Proc, vol. 85, pp. 572-593, 2010.

[3] I. Burazor, M. Imazio, G. Markel, Y. Adler, "Malignant pericardial effusion", Cardiology, vol. 124, pp. 224-232, 2013.

[4] M. M. Refaat, W. E. Katz, "Neoplastic pericardial effusion", Clin Cardiol,vol. 34, pp. 593-598, 2011

[5] R. Schusler, S. L. Meyerson, "Pericardial Disease Associated with Malignancy", Curr Cardiol Rep, vol. 20, pp. 92, 2018. 
[6] M. Imazio, M. Colopi, G. M. De Ferrari, "Pericardial diseases in patients with cancer: contemporary prevalence, management and outcomes", Heart, vol. 106, pp. 569-574, 2020.

[7] E. J. Van Beek, A. H. Stolpen, G. Khanna, B. H. Thompson, "CT and MRI of pericardial and cardiac neoplastic disease", Cancer Imaging, vol. 7, pp. 19-26, 2007.

[8] A. Kumar, A. Puttanna, "Recurrent cardiac tamponade: an initial presentation of lung adenocarcinoma", BMJ Case Rep, 2014, doi: 10.1136/bcr-2013-202553.

[9] M. Chahin, K. Seegobin, S. Maharaj, K. Ramsubeik, "Metastatic ductal adenocarcinoma of the breast presenting with pericardial effusionChallenges in the diagnosis of breast cancer", Clin Case Rep, vol, 7, pp. 2384-2387, 2019.

[10] E. Y. Lee, J. O. Choi, H. N. Park, E. J. Park, G. S. Lee, J. Y. Park, J. S Kim, E. S. Jeon, "Malignant melanoma of unknown primary origin presenting as cardiac metastasis", Korean Circ J, vol. 42, pp. 278-280, 2012.

[11] 11.- Huang JY, Jiang HP, Chen D, Tang HL. Primary gastric signet ring cell carcinoma presenting as cardiac tamponade. World J Gastrointest Oncol. 2011; 3:67-70.

[12] S. Palam, R. Kapoor, A. Abdelkader, J. Kulinski, "Corrigendum to "A Rare Presentation of Cardiac Tamponade from Metastatic Urothelial Carcinoma of the Bladder", Case Rep Cardiol, 2019, doi: $10.1155 / 2019 / 8376360$.

[13] N. Tsoukalas, I. D. Kostakis, S. Demiri, G. Koumakis, V. Barbounis, K. Barbati, A. Efremidis, 'Neoplastic pericarditis as the initial manifestation of a papillary thyroid carcinoma', Ups J Med Sci, vol. 118, pp. 196-198, 2013.

[14] E. Azria, M. Dufeu, P. Fernandez, F. Walker, D. Luton, "Cervical adenocarcinoma presenting as a cardiac tamponade in a 57-year-old woman: a case report", J Med Case Rep, vol. 5, pp. 594, 2011.

[15] G. Liu, Q. Zhang, Z. Li, X. Chen, N. Zhang, J. Zhang, "Endometrial carcinoma complicated by malignant pericardial effusion: A case report on the therapeutic regimen", Medicine (Baltimore), 2019, 98:e17584. doi:10.1097/MD.0000000000017584.

[16] D. C. Lingamfelter, D. Cavuoti, A. C. Gruszecki, "Fata hemopericardial tamponade due to primary pericardial mesothelioma: a case report", Diagn Pathol, vol. 4, pp. 44, 2009.

[17] R. Bussani, F. De-Giorgio, A. Abbate, F. Silvestri, "Cardiac metastases", J Clin Pathol, vol. 60, pp. 27-34, 2007.

[18] A. Tamura, O. Matsubara, N. Yoshimura, T. Kasuga, S. Akagawa, N Aoki, "Cardiac metastasis of lung cancer. A study of metastatic pathways and clinical manifestations", Cancer, vol. 70, pp. 437-442, 1992.

[19] H. Okamoto, T. Shinkai, M. Yamakido, N. Saijo, "Cardiac tamponade caused by primary lung cancer and the management of pericardial effusion”, Cancer, vol. 71, pp. 93-98, 1993. 